\title{
Are dry mergers of ellipticals the way to reconcile model predictions with downsizing?
}

\author{
A. Pipino ${ }^{1}$ and F. Matteucci ${ }^{2,3}$ \\ 1 Astrophysics, University of Oxford, Denys Wilkinson Building, Keble Road, Oxford OX1 3RH, UK \\ e-mail: axp@astro.ox.ac.uk \\ 2 Dipartimento di Astronomia, Universita di Trieste, via G.B. Tiepolo, 11, 34100 Trieste, Italy \\ 3 INAF - Trieste, via G.B. Tiepolo 11, 34100 Trieste, Italy
}

Received 14 January 2008 / Accepted 6 May 2008

\begin{abstract}
Aims. We show that the bulk of the star formation and the galaxy assembly should occur simultaneously in order to reproduce at the same time the downsizing and the chemical properties of present-day massive spheroids within one effective radius.

Methods. By means of chemical evolution models, we create galactic building blocks of several masses and different chemical properties. We then construct a sample of possible merger histories going from a multiple minor merger scenario to a single major merger event aimed at reproducing a single massive elliptical galaxy. We compare our results against the mass- $[\mathrm{Mg} / \mathrm{Fe}]$ and the massmetallicity relations.

Results. We found that a series of multiple dry mergers (no star formation in connection with the merger) involving building-blocks that have been created ad hoc to satisfy the $[\mathrm{Mg} / \mathrm{Fe}]$-mass relation cannot fit the mass-metallicity relation and viceversa. A major dry merger, instead, does not make the agreement with observations worse if it happens between galaxies that already obey both the $\operatorname{mass}(\sigma)-[\mathrm{Mg} / \mathrm{Fe}]$ and the mass $(\sigma)$-metallicity relations. However, this process alone cannot explain the physical reasons for these trends.

Conclusions. Dry mergers alone are not be the way to reconcile the need of a more efficient star formation in the most massive galaxies with the late-time assembly suggested in the hierarchical paradigm to recover the galaxy downsizing.
\end{abstract}

Key words. galaxies: elliptical and lenticular, $\mathrm{cD}$ - galaxies: abundances - galaxies: formation - galaxies: stellar content galaxies: evolution

\section{Introduction}

The scenario describing the formation of elliptical galaxies in the framework of the hierarchical clustering scenario, namely of a major merger involving two spirals at late time (e.g., Kauffmann \& White 1993) has been questioned several times (e.g., Ostriker 1980) since its original formulation. Recent studies (e.g., Thomas \& Kauffmann 1999; Pipino \& Matteucci 2006; Naab \& Ostriker 2007), emphasised a tension between the observed photo-chemical properties and the predicted properties. From the dynamical view point, however, the situation is different. In fact, at least the medium-sized fast-rotating ellipticals (Emsellem et al. 2007) have global morphological and kinematical properties which resemble those of a spiral-spiral merger remnant (e.g., Naab \& Burkert 2003; Cox et al. 2006; Robertson et al. 2006). On the other hand, the most massive ojects are better represented by the outcome of a dissipationless merger (Khochfar \& Burkert 2005; Naab et al. 2006).

From a more general perspective, to reconcile at the same time the anti-hierarchical behaviour of Active Galactic Nuclei (AGNs; e.g., Hasinger et al. 2005), the evolution of luminosity function with redshift (e.g., Bundy et al. 2006), as well as the evidences from the analysis of the stellar populations inhabiting ellipticals (e.g., Thomas et al. 2002), a substantial modification of baryons' behaviour, whith respect to the behaviour of the Dark Matter, seems to be required. In particular, more massive ellipticals are older and form faster with respect to smaller objects (Thomas et al. 2005). This is so-called downsizing (Cowie 1996). Further independent evidence supports the well-known downsizing in the chemical properties of ellipticals, namely the increase of mean stellar $[\mathrm{Mg} / \mathrm{Fe}]$ with galaxy mass (see Matteucci 1994). For instance, the very recent observations of the evolution of the mass-gas metallicity relation with redshift (Maiolino et al. 2008) and the study of the presentday ratio between stars and gas (Calura et al. 2007), both favour the earlier and faster completion of the SF process for the most massive spheroids, with respect to the low-mass spheroids.

Hierarchical modelling, in its latest versions, partly accounts for the downsizing. In pratice, the mass assembly still occurs at late times, but most of the stars have been formed at high redshift in small subunits. The preferred mechanism for the assembly of massive spheroids is a sequence of dry mergers ${ }^{1}$ (e.g., Khochfar \& Burkert 2003; De Lucia et al. 2006). Dry mergers have been observed (Tran et al. 2005; Bell et al. 2006; Rines et al. 2007; and in more extended samples, by van Dokkum 2005), although the criteria used to define a dry-merger observationally have been questioned by, e.g., Donovan et al. (2007). Dry mergers between spheroidal systems are also invoked to explain the so-called boxy ellipticals (e.g., Naab et al. 2006). However, if we restrict ourselves to the most massive elliptical galaxies $\left(L>L_{*}\right)$, they seem to be in place and do not show any signs of significant evolution in mass since $z \sim 1$ (Scarlata et al. 2006; Brown et al. 2007). A great deal of work has been done in the field of dynamical

\footnotetext{
${ }^{1}$ In this paper $d r y$-merger means a pure dissipationless merger of stellar systems, i.e., without any gas and star formation.
} 
simulations, but so far the consequences on the chemical properties of the final stellar populations have not been tested.

On the other hand, in the revised monolithic scenario (Larson 1974; Matteucci 1994; Chiosi \& Carraro 2002; Merlin \& Chiosi 2006) both the mass (or $\sigma)-[\mathrm{Mg} / \mathrm{Fe}]$ (MFMR, hereafter) and the mass (or $\sigma$ )-metallicity (MMR) relations are naturally explained, as shown by Pipino \& Matteucci (2004, PM04 hereafter). In particular, in PM04 for the first time, the inverse wind scenario (Matteucci 1994) plus an initial infall episode, are adopted within a multi-zone formulation. Under reasonable assumptions on the behaviour of the infall timescale and the star formation (SF) efficiency with galactic mass, PM04 showed how this kind of model can reproduce the whole set of chemical and photometric observables simultaneously.

In particular, it is necessary that both the bulk of the star formation and the galactic assembly proceed in lockstep. The same conclusion was reached by Cimatti et al. (2006), who showed that the downsizing trend should also be extended to the mass assembly in the sense that the most massive ellipticals should have assembled before the less massive ones. This conclusion was based on a re-analysis of the rest frame $B$-band COMBO-17 and DEEP2 luminosity functions. The aim of this paper is to show that this has not yet been taken into account in models based on the hierarchical clustering paradigm. We create galactic building blocks of different mass and chemical properties. We then construct a sample of possible merger histories running from a multiple minor merger scenario to a single major merger event aimed at reproducing a single massive elliptical galaxy. We compare the results against the MFMR and the MMR. In the absence of full a dynamical treatment, we cannot undertake a deeper analysis of the mass- and $[\langle\mathrm{Mg} / \mathrm{Fe}\rangle]-\sigma$ relations. In the following, we will refer to the stellar velocity dispersion as a mass tracer, unless otherwise stated.

\section{The model}

The chemical code adopted here is described in PM04 to which we point the reader for more details. In particular, this model is characterised by: Salpeter (1955) initial mass function (IMF); Thielemann et al. (1996), yields for massive stars; Nomoto et al. (1997), yields for type Ia SNe; and van den Hoek \& Groenewegen (1997), yields for low- and intermediate-mass stars (the case with $\eta_{\text {AGB }}$ varying with metallicity).

We will use this model for producing ad hoc progenitors of present-day galaxies to investigate whether the final composite stellar population (CSP) in the merger remnant has properties that match those of observed ellipticals. In particular, for each of them, we list star formation efficiency, infall timescale, and average stellar properties, such as the mass-weighted abundances and abundance ratios (see PM04 for their definition). We define, according to Pagel \& Patchett (1975; see also PM04 and Pipino et al. 2006, hereafter PMC06), the stellar metallicity distribution, $\Upsilon_{\text {prog }}$, as the fraction of stars formed in a given metallicity $(\mathrm{Fe} / \mathrm{H}$ or $\mathrm{Mg} / \mathrm{Fe}$ ) bin.

The possibility to predict $\Upsilon_{\text {prog }}$ properties is important in the context of this study because it allows us to infer the average abundance ratios in the stars. These ratios are useful when we want to compare our theoretical predictions to the observations, often given in terms of SSP-equivalent values, namely luminosity-weighted measures of the properties in the stellar component (see PMC06). The fact that we deal with old objects without any merger-induced SF guarantees that mass-weighted and luminosity-weighted values are very similar in the more

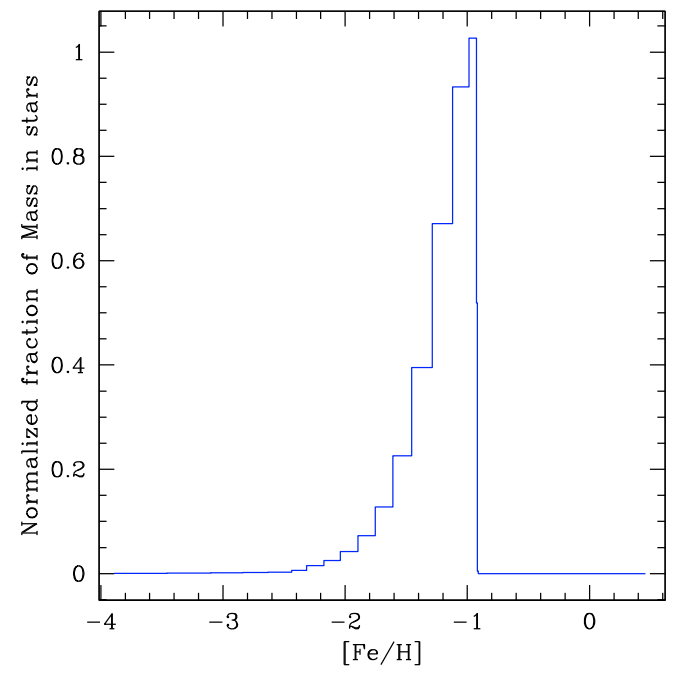

Fig. 1. Predicted stellar metallicity distribution $\Upsilon_{\text {prog }}$ as a function of $[\mathrm{Fe} / \mathrm{H}]$ for the progenitor $\mathrm{A}$.

massive ellipticals (e.g., Arimoto \& Yoshi 1987; Matteucci et al. 1998).

The assumed galactic building blocks have the following characteristics:

A: $\quad$ a $\sim 5 \times 10^{8} M_{\odot}$ building block (luminous mass) that resembles a sort of present-day dwarf spheroidal galaxy (dSph). It has been obtained by assuming a star formation efficiency of $v=0.1 \mathrm{Gyr}^{-1}$, while the infall timescale is $\tau=0.5 \mathrm{Gyr}$ in agreement with the prescriptions of Lanfranchi \& Matteucci $(2004,2007)$. For this model, we predict $[\langle\mathrm{Mg} / \mathrm{Fe}\rangle]=0.28 \mathrm{dex}$ and $[\langle\mathrm{Fe} / \mathrm{H}\rangle]=-1.2 \mathrm{dex}$, and a stellar metallicity distribution function $\Upsilon_{\text {prog }}$ shown in Fig. 1. The star formation in this block lasts $2.4 \mathrm{Gyr}$, which is the time at which a supernovae-driven galactic wind occurs.

A+: $a \sim 5 \times 10^{8} M_{\odot}$ building block has been obtained by assuming a higher star formation efficiency with respect to the previous case of $v=10 \mathrm{Gyr}^{-1}$, while the infall timescale is $\tau=0.5 \mathrm{Gyr}$.

For this model, we predict $[\langle\mathrm{Mg} / \mathrm{Fe}\rangle]=0.57 \mathrm{dex}$ and $[\langle\mathrm{Fe} / \mathrm{H}\rangle]=-0.9$ dex. The star formation here lasts only $130 \mathrm{Myr}$ due to the faster occurrence of a galactic wind.

B: $\quad$ a $\sim 5 \times 10^{8} M_{\odot}$ building block has been obtained by assuming a star formation efficiency of $v=1 \mathrm{Gyr}^{-1}$, while the infall timescale is $\tau=3 \mathrm{Gyr}$.

For this model, we predict $[\langle\mathrm{Mg} / \mathrm{Fe}\rangle]=-0.1$ dex and $[\langle\mathrm{Fe} / \mathrm{H}\rangle]=0.63$ dex, and a stellar metallicity distribution $\Upsilon_{\text {prog }}$ function shown in Fig. 2. The star formation here lasts $1.8 \mathrm{Gyr}$.

E: $\quad a \sim 10^{11} M_{\odot}$ elliptical galaxy that matches both the MMR and the MFMR as well as the colour-magnitude relation (CMR, Bower et al. 1992). We refer to PM04's model IIb for the same mass. For this model, we predict an overall $[\langle\mathrm{Mg} / \mathrm{Fe}\rangle]=0.25 \mathrm{dex}$ and $[\langle\mathrm{Fe} / \mathrm{H}\rangle]=0.04$ dex. The stellar metallicity distribution is presented in Fig. 3 (solid line). We refer the reader to PMC06 for a more detailed description of the stellar metallicity distributions $\Upsilon_{\text {prog }}$ for such a model.

The final massive elliptical (F) we want to simulate is a $\sim 2 \times$ $10^{11} M_{\odot}$ galaxy. In particular, we expect it to have $[\langle\mathrm{Mg} / \mathrm{Fe}\rangle]=$ 0.27 dex according to PM04's model IIb predictions for the same mass. 


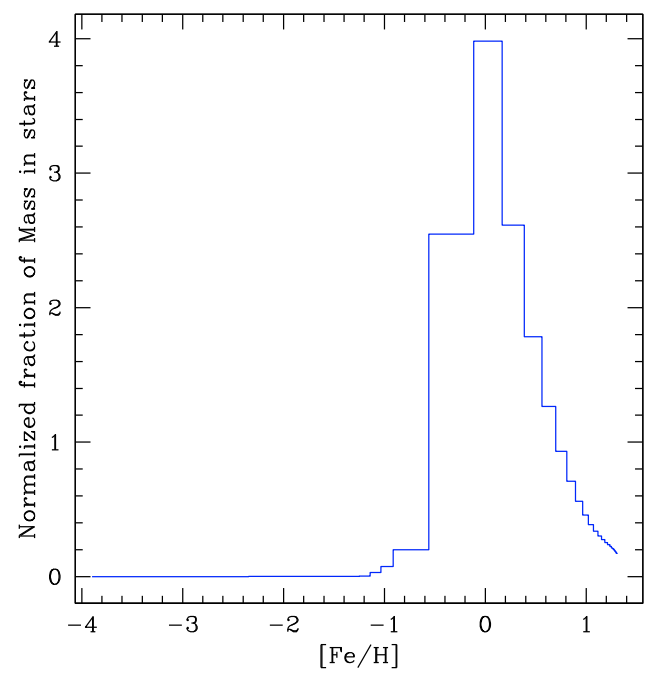

Fig. 2. Predicted stellar metallicity distribution $\Upsilon_{\text {prog }}$ as a function of $[\mathrm{Fe} / \mathrm{H}]$ for the progenitor $\mathrm{B}$.

For the sake of simplicity, we will assume that our model galaxies do not have radial gradient in $\alpha$-enhancement. As shown by Pipino et al. (2008), in fact, even though most ellipticals form outside-in, the expected strong and positive $[\langle\alpha / \mathrm{Fe}\rangle]$ gradient can be affected by the metal rich gaseous flows inside the galaxy acting together with the SFR. We also recall that observations suggest that the observed gradient slope in the $[\langle\mathrm{Mg} / \mathrm{Fe}\rangle]$ has a null mean value (e.g., Mehlert et al. 2003). Therefore, we will refer to a one-zone model in which the metallicity and the $\alpha$ enhancement do not vary with radius. In general, we will expect that mergers cannot account for the steep metallicity gradient observed in the majority of ellipticals (e.g., Carollo et al. 1993), and we postpone to a forthcoming paper the analysis of the gradients survival to several dry-mergers.

Under these assumptions, we predict the properties of the CSP of the merger remnant in a straightforward manner. In fact, the stellar metallicity distribution function for the end product of a dry-merger is simply $\Upsilon_{\text {final }}$ summed over all progenitors and can be written as:

$\Upsilon_{\text {final }}=\sum_{\text {prog }} M_{*, \text { prog }} \cdot \Upsilon_{\text {prog }}(t, Z) / \sum_{\text {prog }} M_{*, \text { prog }}$,

where $M_{* \text {,prog }}$ is the stellar mass of the single progenitors. Similar equations hold for other distributions as functions of either $[\mathrm{Mg} / \mathrm{Fe}]$ or $[\mathrm{Fe} / \mathrm{H}]$. We stress again that this is possible because we are studying dry merger remnants, i.e., systems where no further SF is allowed to occur.

We also emphasise that we present an exercise whose assumptions are rather extreme (i.e., galaxies formed only via dry mergers) and without taking into account the observed merger rate and its relation with redshift, environment, and possibly the morphology of the progenitors (e.g., Lin et al. 2008).

\section{Results and discussion}

\subsection{Multiple dry mergers of equal progenitors}

Let us first assume the extreme case in which our massive elliptical has been made by merging of several progenitors of the kind $A$ only, as expected from galaxy formation models that assume a short SF process at high redshift, but let the galaxy assembly happen much later (e.g., De Lucia et al. 2006; Kobayashi et al. 2007).

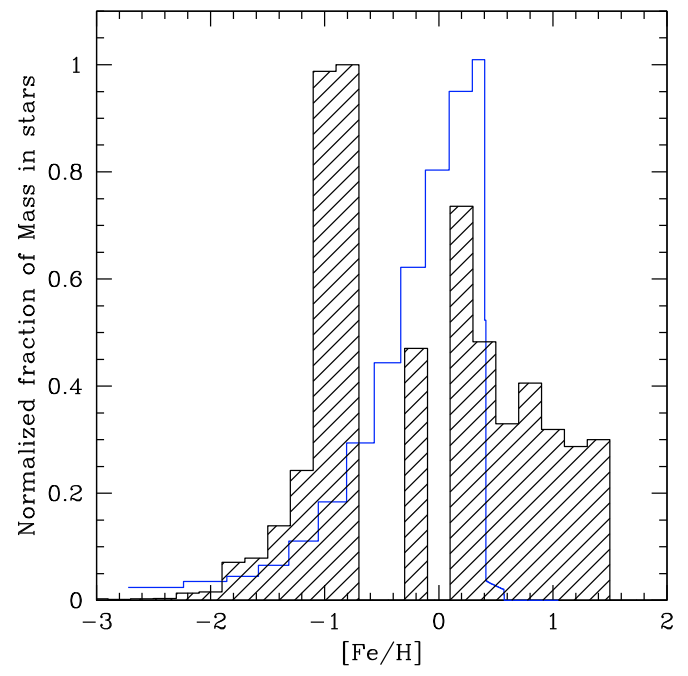

Fig. 3. Shaded hystogram: predicted stellar metallicity distribution $\Upsilon_{\text {final }}$ as a function of $[\mathrm{Fe} / \mathrm{H}]$ for the case in which $50 \%$ of the final massive galaxy is made of progenitor A-like building blocks and the rest of progenitors B. For comparison, the prediction for the $\Upsilon_{\text {prog }}$ of the PM04 best model (case E) is presented (solid line).

In order to have the right final mass, we need 400 of such small building blocks. Since progenitor A has been built to yield the final correct $\alpha$ enhancement, our massive spheroid will have a $\mathrm{Mg}$ enhancement of 0.28 dex and will match the average observational value for galaxies of the same mass fairly well. We can infer from Eq. (1) that the final stellar metallicity distribution will still look like Fig. 1, therefore its final metallicity in terms of $[\mathrm{Fe} / \mathrm{H}]$ will remain very low, thus not matching either the MMR or the CMR.

The predicted value of the SFR per unit mass is $0.02 / \mathrm{Gyr}$ for the progenitor of kind $A$. Again, we can infer that the final spheroid will have the same value, at variance with the results from Thomas et al. (2005), which require this factor to be at least 2-3/Gyr, namely a factor of one hundred higher. Such a high SFR is needed also to reproduce the observed SFR in Lyman Break ( 5-940 $h^{-2} M_{\odot} \mathrm{yr}^{-1}$, Shapley et al. 2001) and SCUBA (e.g., Swinbank et al. 2004) galaxies.

On the other hand, a quasi-monolithic model can naturally have the required SFR per unit mass. In fact, the prediction by PM04 (Fig. 4) is in good agreement for what concerns shape, timescale, and mean redshift of formation with those inferred by Thomas et al. (2005, see their Fig. 10), the only difference being the sharp truncation due to the galactic wind. It should be noted that many models based on the hierarchical clustering, which claim to have incorporated downsizing (e.g., De Lucia et al. 2006; Kobayashi et al. 2007), have an average SFR per unit mass lower at least by a factor of 3-5 than what is required from chemical evolution studies and line-strenght indices analysis to reproduce the $[\mathrm{Mg} / \mathrm{Fe}]$ in massive ellipticals; according to our calculations, with such a low SFR per unit mass is possible to reproduce only a very modest $\alpha$-enhancement (if any). In fact, such a value for the SFR per unit mass $(\sim 1 / \mathrm{Gyr})$ will only suffice to explain the $[\langle\mathrm{Mg} / \mathrm{Fe}\rangle] \sim 0.1 \mathrm{dex}$ of the less massive spheroids.

We tried to overcome the problem of having a too low SFR by introducing another ad hoc building block, namely the progenitor $A+$, which has a SFR per unit mass of the order of unity. In this case, however, the lack of agreement with the MMR is much more evident, and also the predicted $[\langle\mathrm{Mg} / \mathrm{Fe}\rangle]$ is too high. We note in passing that if we allow for a subsequent gas-rich 


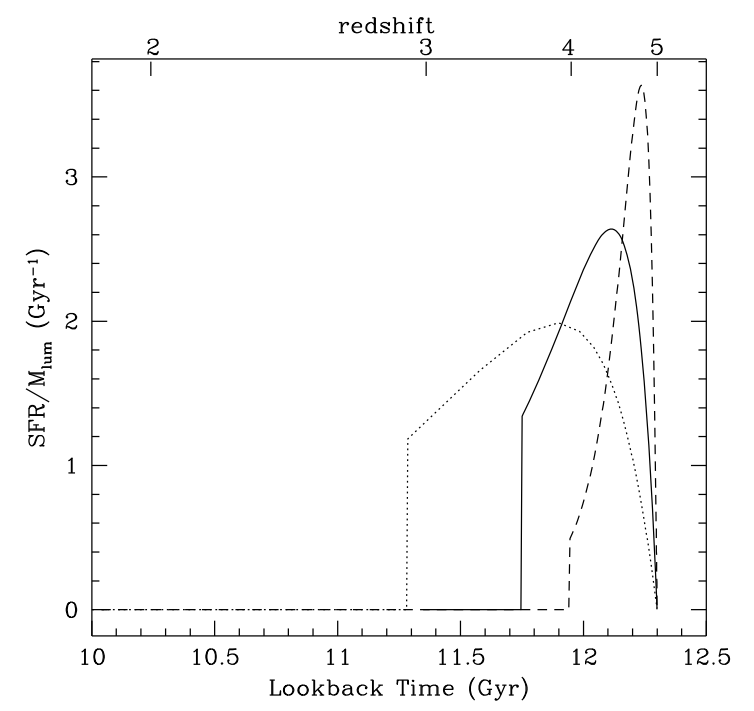

Fig. 4. Predicted SFR per unit mass for several masses, PM04's best model. The model $\mathrm{E}$ is represented by a solid line.

merger triggering a substantial episode of SF, we may be able to reproduce the MFMR, but we fail in obtaining other properties, such as the CMRs or the MMR, as shown by Pipino \& Matteucci (2006, see, e.g., the models discussed in their Sect. 3.3).

On the other hand, there can exist the extreme case in which the galaxy is created by several progenitors of the kind $B$. The results of this analysis show that this model can reproduce the MMR, but it predicts an underabundance of $\alpha$-elements relative to $\mathrm{Fe}$, at variance with observations.

We conclude that we cannot form massive spheroids from a sequence of several dry mergers between building blocks of the same kind (similar mass and chemical properties), even if the progenitors are chosen to have the correct $\alpha$-enhancement. For the same reason, present-day, low-mass ellipticals, which satisfy both the MMR and the MFMR, cannot be the building blocks of massive ones. This conclusion can be extended to the CMR, since the colour differences are mainly driven by metallicity. Remarkably, similar conclusions have been obtained by Ciotti et al. (2007) by studying the dynamical properties of ellipticals. Therefore, pure dissipationless merger of similar stellar systems cannot change the metallicity, the $\alpha$-enhancement, the colours, and the virial velocity dispersion.

\subsection{Multiple dry mergers of different progenitors}

Now we relax the extreme assumption of the previous section and allow for two or more kinds of progenitors for our massive elliptical. If, for simplicity sake, there is a fraction $f=$ $50 \%$ of the final mass coming from progenitors of the type A and $1-f=50 \%$ from progenitors $\mathrm{B}$, the final stellar metallicity distribution (shaded hystogram in Fig. 3) will be closer to the one expected for a normal elliptical (solid line in Fig. 3)and the outcome will match the CMR and the MMR, being its final $[\langle\mathrm{Fe} / \mathrm{H}\rangle]=0.66 \mathrm{dex}$, but the predicted $[\langle\mathrm{Mg} / \mathrm{Fe}\rangle]=0.06 \mathrm{dex}$ is still too low. Moreover, this scenario cannot represent a solution for the still too low SFR per unit mass.

If we repeat the same exercise with model $A+$ and $\mathrm{B}$, these latter quantities agree better with the values inferred from the observations, and we also notice an improvement for $[\langle\mathrm{Mg} / \mathrm{Fe}\rangle]$, which now amounts to 0.1 dex.
We admit that the portion of the parameter space that we are investigating is quite small - although the choice of the models is sensible - the main aim of this investigation being the study of a few clear and extreme cases. Such examples serve to probe to what extent the random nature of the merger process models can be accommodated within the observational uncertainties. A more comprehensive analysis featuring a proper merger history based on the hierachical growth of structure and a self-consistent chemical evolution is in preparation.

However, even in the case in which either two more suitable progenitors can be found, or a different mixture of several progenitors can predict the right final chemical properties for a given final galactic mass, several questions arise: i) why are only dSph (i.e., progenitor A) still observable in the local universe? (but see Robertson et al. 2005); ii) why is the fraction $f$ such that none of the two classes is predominant?; and iii) since the $[\langle\mathrm{Mg} / \mathrm{Fe}\rangle]$ correlates with the final galactic mass, one should expect progenitors with different initial (i.e., pre-merger) properties, which scale accordingly to the final mass of the object, to live in the early universe. How it is possible that they know in advance what they are about to build later on? Finally, even if a selection mechanism is at work and it leads to an agreement between model and observed chemical properties, it must be able to account for other scaling relations, such as the Faber-Jackson (Faber \& Jackson 1976) and the Kormendy (1977) relations as well as the Fundamental Plane (e.g., Dressler et al. 1987).

It is also interesting to note that Bournaud et al. (2007) claimed that repeated minor mergers - such as the ones studied in this section - can theoretically form massive boxy elliptical galaxies without major mergers, being more frequent than the latter, in particular at moderate redshifts. The mechanism put forward by Bournaud et al. (2007) could explain the morphology and the dynamical properties of the merger remnant; in particular, it might be a viable alternative to overcome the issues in the major-merger scenario (Naab \& Ostriker 2007) to explain the high boxiness of massive ellipticals. Unfortunately, Bournaud et al. (2007) explored only too narrow a mass range to understand whether their argument helps in reproducing the observed scaling relations for elliptical galaxies.

\subsection{Multiple minor-dry merger on an already formed elliptical galaxy}

At variance with the previous section, we now test the scenario in which the final galaxy is built via a series of a minor dry mergers, namely adding several progenitors of either type (A-like or B-like) to a galaxy like progenitor $\mathrm{E}$ until we double its mass (therefore, we need roughly 200 small building blocks). Since the model E stars contain the right amount of $\alpha$-enhancement and mean metallicity, this case will help us in assessing whether the accretion of several progenitors of either types A or B can worsen the agreement with observations. Following the same line of reasoning as in the previous sections, we first assume that we want to build the final galaxy as the sum of E and only type $\mathrm{A}+$ progenitors. We obtain the $\Upsilon_{\text {final }}$ for model $\mathrm{F}$ according to Eq. (1), and we find that the final $[\langle\mathrm{Mg} / \mathrm{Fe}\rangle]=0.26$ does not exceed the observational boundaries. If we assume that $F$ is the result of a progenitor like $\mathrm{E}$ plus roughly two hundreds building blocks of the type $\mathrm{B}$, we predict $[\langle\mathrm{Mg} / \mathrm{Fe}\rangle]=0.13$, which is on the lower observational boundary (see Fig. 5, upper panel). The two cases presented above bracket a region of the paramenter space in which the final galaxy F can be obtained through a series of dry mergers involving a galaxy like $\mathrm{E}$ and progenitors like A, $\mathrm{A}+$, and $\mathrm{B}$, in different mixtures. Three main conclusions can 

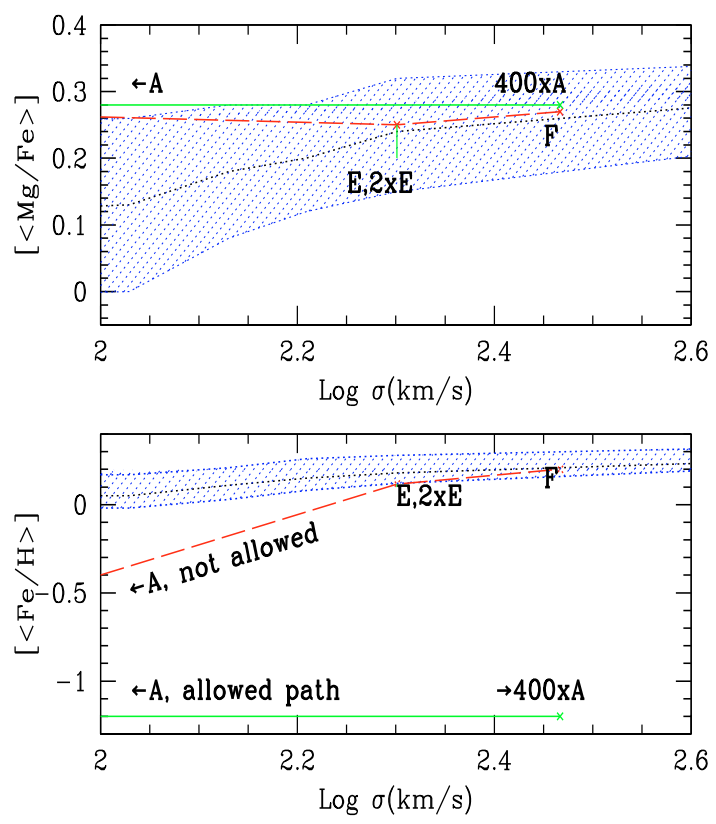

Fig. 5. Predicted evolutionary paths (solid: allowed by the analysis of the chemical properties; dashed: not allowed, see text) in the $[\langle\mathrm{Mg} / \mathrm{Fe}\rangle]-$ $\sigma$ (upper panel) and $[\langle\mathrm{Fe} / \mathrm{H}\rangle]-\sigma$ (lower panel) planes. The initial, intermediate, and final points are marked according to the galaxy (progenitor) type and the merger end-product, respectively. The shaded areas encompass the observations (Nelan et al. 2006), whose average trends with the velocity dispersion are given by the black dotted lines.

be drawn from a scenario featuring multiple small progenitors accreted by a medium-sized ellipticals until it doubles its mass: i) multiple minor dry mergers cannot be ruled out on the basis of current observations, but they are only small perturbations for a massive elliptical which formed monolithically; ii) they may explain the observed scatter in the $[\langle\mathrm{Mg} / \mathrm{Fe}\rangle]$ values at a given galactic mass, although it can also be explained in the framework of the revised monolithic scheme by small differences in either the star formation efficiency or the infall timescale with respect to the PM04 best value (tuned to represent the average galaxy); and iii) in any case, they cannot explain the trend of $[\langle\mathrm{Mg} / \mathrm{Fe}\rangle]$ with $\sigma$, because, even if they may lead to a modest increase in $[\langle\mathrm{Mg} / \mathrm{Fe}\rangle]$, the stellar velocity dispersion does not increase (Ciotti et al. 2007).

It is obvious that a few events like the ones depicted in this section can occur, for instance, in a dense environment such as a cluster of galaxies where a residual ongoing SF is also detected in massive ellipticals and cD (Bildfell et al. 2008). The amount of SF inferred from UV spectra (Kaviraj et al. 2007) from redshift 1 to the present-day (and consistent with the merger rate by Khochfar \& Burkert 2006) will lead to a modest increase (1-5 percent) in the stellar mass, even tough no SNII explosions have been detected to-date (Mannucci et al. 2007, but they might still occur in S0; e.g., Pastorello et al. 2007); therefore, we consider it either as an accident or a consequence of the environment, rather than a signature of any particular galaxy formation scenario. In fact, according to our previous calculations (Pipino \& Matteucci 2006), such a low-intensity late SF episode cannot significantly lower the $[\langle\mathrm{Mg} / \mathrm{Fe}\rangle]$ ratio.

Interestingly, recent observational evidences (Daddi et al. 2005; Trujillo et al. 2007) favour an increase in the size ellipticals by a factor of about 4 since redshift $z=1$, plausibly associated with the occurrence of dry mergers. However, the simulations show that this kind of accretion occurs mainly outside one effective radius (Naab et al. 2007) and it might be due to accretion of small satellites (Daddi et al. 2005); therefore, we do not expect them to affect the properties of the galactic core, whose stars obey the MMR and the MFMR. Again, even in the framework of the revised-monolithic scenario, these episodes are unavoidable either in a dense environment or considering the fact the massive ellitpicals are 12 Gyr old. Therefore, they had enough time to interact with their satellites.

\subsection{Late major dry merger}

Finally, we want to test the feasibility of a major dry merger between two massive spheroids of the kind $E$ to produce the galaxy $F$. In this case, the final elliptical will double its mass and keep a mean $[\langle\mathrm{Mg} / \mathrm{Fe}\rangle]=0.25 \mathrm{dex}$, which does not differ much from the value expected by model F. Nevertheless, a problem arises from the fact that, for a pure dissipationless merger between two objects of equal mass and same velocity dispersion $\sigma$, the final object will double its mass, but preserve $\sigma$ (Ciotti et al. 2007). Therefore, we cannot move along the direction of the observed $[\langle\mathrm{Mg} / \mathrm{Fe}\rangle]-\sigma$ relation. This also seems to be the case when the stellar central velocity dispersion is allowed to (modestly) increase due to non-homology effects (Nipoti et al. 2003). We stress that this clear and straightforward consequence of the virial theorem is often neglected in works that aim at reconciling the prediction from the hierarchical clustering scenario with the evidences coming from the chemistry.

Nonetheless, we exploit the $[\langle\mathrm{Mg} / \mathrm{Fe}\rangle]$-mass relation to infer some constraints on the number of major dry mergers involving massive spheroids. From Thomas et al. (2005), we know that $[\langle\mathrm{Mg} / \mathrm{Fe}\rangle]=-0.459+0.062 \log \left(M_{*}\right)$ with an intrinsic scatter of \pm 0.05 dex. This means that an elliptical galaxy that satisfies the average trend, can undergo either $\sim 2-4$ major dry mergers (if only one has mass ratio $1: 1$, the rest being either $1: 2$ or $1: 3$ ) or $\sim 2-3$ major dry-mergers (if the mass ratio is always $1: 1$, i.e., in the first step, we create two galaxies, each one via a merger of two units like model E $(2 \mathrm{xE})$, and then we let them merge together), before crossing the boundary set by observations. Remarkably, such a limit is in agreement with recent observational estimates for the average number of major mergers experienced by elliptical galaxies since redshift 1.2 (e.g., Lin et al. 2008). Unfortunately, the nature of our exercise does not allow us either to put precise constraints or to make predictions on the merger rate. However, if we take into account the fact that the dry-merger rate seems to decline quite strongly with redshift, we may argue that the maximum major-merger rate allowed by the MFMR is 0.2-0.3/Gyr in agreement with Bell et al. (2006)'s estimate. In any case, following the same argument of the previous sections, no arbitrary sequence of dry mergers of a model $\mathrm{E}$ galaxy with similar progenitor can ever form a $10^{12} M_{\odot}$ spheroid with the highest observed $[\langle\mathrm{Mg} / \mathrm{Fe}\rangle]$ for that mass.

\section{Conclusions}

Before drawing our final remarks, we summarise the results obtained in the studied case by means of Fig. 5, where the average $[\langle\mathrm{Mg} / \mathrm{Fe}\rangle]$ and the average $[\langle\mathrm{Fe} / \mathrm{H}\rangle]$ are plotted versus $\log (\sigma)$. In this figure, we sketch the allowed paths as solid lines whereas we plot as dashed lines what is impossible on the basis of stellar population arguments. We compare them with the observations by Nelan et al. (2006): the black dotted line being the mean value, and the shaded area brackets the observational scatter reported by the authors. These values are in agreement with the previous analysis by Thomas et al. (2005), although in 
Nelan et al. (2006) the slope of the MFMR is somewhat flatter at high velocity dispersions.

As done in the previous sections, we follow the merger history that links progenitors $A$ to the intermediate stage $E$ to the massive elliptical $F$ as an example of a possible accretion history, which may lead to the creation of a massive spheroid from small subunits. With $400 x A$, we name the outcome of the multiple mergers described in Sect. 3.1. We already know that some of the chemical properties of such a galaxy will differ from those featured by model F. We assume that the progenitors of type A inhabit the upper (lower) left corner in the upper (lower) panel, without any further specification on their initial $\sigma$ and on the dynamical outcome of the multiple mergers. To be conservative, in fact, we assume that they can somehow increase their $\sigma^{2}$, but we recall that, where the build-up of model 400xA a sequence of pure parabolic mergers ${ }^{3}$ between collisionless systems, its final $\sigma$ would be equal to the maximum value of the stellar velocity dispersion among the progenitors (Ciotti et al. 2007; see also Nipoti et al. 2003, for the effects of non-homology). This means that the remnant merger would probably lie very close to $A$ in the upper panel of Fig. 5, without moving leftward as we assume in our simple sketch. From the upper panel of Fig. 5, we notice that the model $400 \mathrm{xA}$ is fairly close to the model that we want to reproduce through the sequence of mergers $(\mathrm{F})$. Therefore, we mark this path (solid line) in the $[\mathrm{Mg} / \mathrm{Fe}]-\sigma$ plane as allowed, meaning that, on a pure chemical evolution basis, it is possible to create a massive spheroid, which satisfies the MFMR starting from $\alpha$-enhanced building blocks. On the other hand, the lower panel of Fig. 5 tells us that the galaxy of type 400xA falls short in reproducing the MMR by more than two orders of magnitude. A path joining $A$ with $E$ and $F$ (dashed line) is impossible if we want to satisfy both the observed relationships. We do not show here the paths leading to a final $\mathrm{F}$ galaxy from building blocks of type B. It is intuitive that they will be allowed on the metallicity- $\sigma$ plane, but they will be outside the region delimited by the observations in the $[\mathrm{Mg} / \mathrm{Fe}]-\sigma$ plane. If we allow galaxies to form through a sequence of mergers involving different progenitors, given the random nature of the hierarchical assembly, it is natural to expect a scatter in the predicted MMR and the MFMR much larger than the observed ones and no slope at all. We also expect this to happen in the most recent models based on the hierarchical assembly, and even if they features several gestures toward the downsizing, as it has indeed been shown by Nagashima et al. (2005).

This fact and our previous works on the quasi-monolitic formation of massive ellipticals (PM04) as well as the effect of major wet mergers (Pipino \& Matteucci 2006) allow us to derive the first important conclusions:

i) the diagnostic power of the MMR and MFMR relies on the fact that the mechanisms required to satisfy the former tend to worsen the agreement with the latter, and viceversa;

ii) only when most of the star formation process and the galactic assembly occur at roughly the same time and the same place can both relations be fulfilled.

As expected from the discussion of Sect. 3.4, the outcome (called $2 x E$ in Fig. 5) of a major dry merger involving two massive

\footnotetext{
2 This might happen if the progenitors are already bound to each other, as in the case in which they inhabit the same massive Dark Matter halo. However, the energy must have the exact value for the merger endproduct to obey to the Fundamental Plane and therefore this case seems rather unlikely.

3 This seems to be the case for nearly $50 \%$ of the merger events (Khochfar \& Burkert 2006)
}

ellipticals, will occupy the same place of its progenitor $E$ and does not move to where it is expected (i.e., at $F$ ), therefore we mark this path as not allowed.

Moreover, the model $E$ has been designed to represent an average elliptical of medium size, although the observations tell us that there are galaxies of the same mass, but lower $\alpha$ enhacement (as low as 0.1 dex, lower boundary of the shaded region - Fig. 5, upper panel). This means that, in principle, we should also be able to observe giant ellipticals with $[\langle\mathrm{Mg} / \mathrm{Fe}\rangle]$ as low as $\sim 0.1$ dex. Since this is not the case, we are not only in need of a way to increase $\sigma$ without having any substantial wet mergers (and further SF), but also in need of a way to invoke some selective mergers. Other constraints will come from the study of the joint evolution of the MFMR and MMR with redshift.

In conclusion, it seems hard to reproduce all giant ellipticals either via a pure sequence of multiple minor dry mergers, or via major dry mergers. However, the scatter of the MFMR is such that the occurrence of 1-3 major-dry mergers during the galactic lifetime cannot be ruled out and, indeed, it is in agreement with the observations (e.g., Bell et al. 2006). For the same reason, several accretion episodes of small satellites onto a massive "monolithic" elliptical galaxy, can be classified as perturbations that may help explain the observational scatter. Moreover, it is still to be understood whether a mixed (namely including both wet and dry mergers) model that tracks the observed evolution of the merger rates amongst different galactic morphologies (e.g., Lin et al. 2008) can overcome the above difficulties ${ }^{4}$. This is beyond the scope of the present paper, which (along with PM06) aims at being only a first step into a quantitative analysis of the chemical properties of merger remnants. We stress that we present some rather extreme cases (i.e., galaxies formed only via dry mergers) as opposed to PM06 where we presented only wet mergers. Results by a semi-analytical model, which incorporates several gestures toward the reproduction of the downsizing as well as the galactic colour bimodality, with a full and selfconsistent treatment of the chemical evolution, will be presented in a forthcoming paper (Pipino et al., in preparation).

Acknowledgements. We acknowledge useful discussions with T. Naab. We thank the referee for the careful reading. L. Ciotti is warmly thanked for a timely reading of the paper and many enlightening comments.

\section{References}

Arimoto, N., \& Yoshii, Y. 1987, A\&A, 173, 23

Bell, E. F., Naab, T., McIntosh, D. H., et al. 2006, ApJ, 640, 241

Bildfell, C., Hoekstra, H., Babul, A., \& Mahdavi, A. 2008 [arXiv: 0802 . 2712]

Bournaud, F., Jog, J., \& Combes, F. 2007, A\&A, 476, 1179

Bower, R. G., Lucey, J. R., \& Ellis, R. S. 1992, MNRAS, 254, 589

Brown, M. J. J, Dey, A., Jannuzi, B. T., et al. 2007, ApJ, 654, 858

Bundy, K., Ellis, R. S., \& Conselice, C. J. 2005, ApJ, 625, 621

Calura, F., Jimenez, R., Panter, B., Matteucci, F., \& Heavens, A. F. 2007, ApJ, in press [arXiv:0707.1345]

Carollo, C. M., Danziger, I. J., \& Buson, L. 1993, MNRAS, 265, 553

Cimatti, A., Daddi, E., Renzini, A., et al. 2004, Nature, 430, 184

Cimatti, A., Daddi, E., \& Renzini, A. 2006, A\&A, 453, 29

Ciotti, L., Lanzoni, B., \& Volonteri, M. 2007, ApJ, 658, 65

Cox, T. J., Dutta, S. N., Di Matteo, T., et al. 2006, ApJ, 650, 791

Cowie, L. L., Songaila, A., Hu, E. M., \& Cohen, J. G. 1996, AJ, 112, 839

Daddi, E., Renzini, A., Pirzkal, N., et al. 2005, ApJ, 626, 680

De Lucia, G., Springel, V., White, S. D. M., Croton, D., \& Kauffmann, G. 2006, MNRAS, 366, 499

\footnotetext{
4 Thomas \& Kauffmann (1999) already showed that this is not the case for the class of earlier hierarchical models not taking into account the downsizing.
} 
Donovan, J. L., Hibbard, J. E., \& van Gorkom, J. H. 2007 [arXiv: 0706 . 0734] Dressler, A., Lynden-Bell, D., Burnstein, D., et al. 1987, ApJ, 313, 42 Emsellem, E., Cappellari, M., Krajnovic', D., et al. 2007, MNRAS, 379, 401 Faber, S. M., \& Jackson, R. E. 1976, ApJ, 204, 668

Hasinger, G., Miyaji, T., \& Schmidt, M. 2005, A\&A, 441, 417

Kauffmann, G., \& White, S. D. M. 1993, MNRAS, 261, 921

Kaviraj, S., Peirani, S., Khochfar, S., Silk, J., \& Kay, S. 2007 [arXiv:0711.1493]

Khockfar, S., \& Burkert, A. 2003, ApJ, 597L, 117

Khockfar, S., \& Burkert, A. 2005, MNRAS, 359, 1379

Khockfar, S., \& Burkert, A. 2006, A\&A, 445, 403

Kobayashi, C., Springel, V., \& White, S. D. M. 2007, MNRAS, 376, 1465

Kormendy, J. 1977, ApJ, 218, 333

Lanfranchi, G. A., \& Matteucci, F. 2004, MNRAS, 351, 1338

Lanfranchi, G. A., \& Matteucci, F. 2007, A\&A, 468, 927

Larson, R. B. 1974, MNRAS, 166, 585

Maiolino, R., et al. 2008, to appear on the proceedings of A Century of Cosmology: Past, Present and Future [arXiv:0712 .2880]

Matteucci, F. 1994, A\&A, 288, 57

Matteucci, F., Ponzone, R., \& Gibson, B. K. 1998, A\&A, 335, 855

Mannucci, F., Maoz, D., Sharon, K., et al. 2007, MNRAS, tmp, 1132

Mehlert, D., Thomas, D., Saglia, R. P., Bender, R., \& Wegner, G. 2003, A\&A, 407, 423

Merlin, E., \& Chiosi, C. 2006, A\&A, 457, 437

Naab, T., \& Ostriker, J. P. 2007 [arXiv:astro-ph/0702535]

Naab, T., Khochfar, S., \& Burkert, A. 2006, ApJ, 636, L81

Naab, T., Johansson, P. H., Ostriker, J. P., \& Efstathiou, G. 2007, ApJ, 658, 710

Nagashima, M., Lacey, C. G., Okamoto, T., et al. 2005, MNRAS, 363L, 31

Nelan, J. E., Smith, R. J., Hudson, M. J., et al. 2005, ApJ, 632, 137

Nipoti, C., Londrillo, P., \& Ciotti, L. 2003, MNRAS, 342, 501
Nomoto, K., Hashimoto, M., Tsujimoto, T., et al. 1997, Nucl. Phys. A, 621, 467 Ostriker, J. P. 1980, ComAp, 8, 177

Pastorello, A., Della Valle, M., Smartt, S. J., et al. 2007, Nature, 449, 1

Pagel, B. E. J., \& Patchett, B. E. 1975, MNRAS, 172, 13

Pipino, A., \& Matteucci, F. 2004, MNRAS, 347, 968 (PM04)

Pipino, A., \& Matteucci, F. 2006, MNRAS, 365, 1114

Pipino, A., Matteucci, F., \& Chiappini, C. 2006, ApJ, 638, 739 (PMC06)

Pipino, A., D'Ercole, A., \& Matteucci, F. 2008, A\&A, 484, 679

Rines, K., Finn, R., \& Vikhlinin, A. 2007 [arXiv:0708.0011]

Robertson, B., Bullock, J. S., Font, A. S., Johnston, K. V., \& Hernquist, L. 2005, ApJ, 632, 872

Robertson, B., Cox, T. J., Hernquist, L., et al. 2006, ApJ, 641, 21

Salpeter, E. E. 1955, ApJ, 121, 161

Scarlata, C., Carollo, C. M., Lilly, S. J., et al. 2007, ApJS, 172, 494

Shapley, A. E., Steidel, C., Adelberger, K. L., et al. 2001, ApJ, 562, 95

Swinbank, A. M., Smail, I., Chapman, S. C., et al. 2004, ApJ, 617, 64

Thielemann, F. K., Nomoto, K., \& Hashimoto, M. 1996, ApJ, 460, 408

Thomas, D., \& Kauffmann, G. 1999, in Spectroscopic dating of stars and galaxies, ed. I. Hubeny, S. Heap, \& R. Cornett, ASP Conf. Ser., 192, 261

Thomas, D., Maraston, C., \& Bender, R. 2002, Ap\&SS, 281, 371

Thomas, D., Maraston, C., Bender, R., \& Mendes de Oliveira, C. 2005, ApJ, 621, 673

Thornton, K., Gaudlitz, M., Janka, H.-T., \& Steinmetz, M. 1998, ApJ, 500, 95 Trager, S. C., Faber, S. M., Worthey, G., \& Gonzalez, J. J. 2000, AJ, 119, 1654 Tran, K.-V. H., van Dokkum, P., Franx, M., et al. 2005, ApJ, 627, L25 Trujillo, I., Conselice, C. J., Bundy, K., et al. 2007, MNRAS, 382, 109 van den Hoek, L. B., \& Groenewegen, M. A. T. 1997, A\&AS, 123, 305 van Dokkum, P. G. 2005, AJ, 130, 2647

Worthey, G., Faber, S. M., \& Gonzalez, J. J. 1992, ApJ, 398, 69 